\title{
Screening Potential Prognostic Factors for gastric carcinoma and Indicators for Tumor Microenvironment Remodeling in Female and Male patients Based on TCGA Data Mining
}

\section{Xinghua Hai}

First Teaching Hospital of Tianjin University of Traditional Chinese Medicine

\section{Fang Liu}

First Teaching Hospital of Tianjin University of Traditional Chinese Medicine

\section{Li Jin}

First Teaching Hospital of Tianjin University of Traditional Chinese Medicine

\section{Xiaoyu Wang}

First Teaching Hospital of Tianjin University of Traditional Chinese Medicine

\section{Yan Pang}

First Teaching Hospital of Tianjin University of Traditional Chinese Medicine

Huanan Li

First Teaching Hospital of Tianjin University of Traditional Chinese Medicine

\section{Wei Zhang}

First Teaching Hospital of Tianjin University of Traditional Chinese Medicine

\section{Xiongfei Luo}

First Teaching Hospital of Tianjin University of Traditional Chinese Medicine

Qing Sun ( $\sim$ 13820290606@163.com )

First Teaching School of Tianjin University of Traditional Chinese Medicine

\section{Research article}

Keywords: Gastric carcinoma, FCGR2A, GFRA1, gender, tumour microenvironment, tumour-infiltrating immune cell

Posted Date: February 26th, 2021

DOl: https://doi.org/10.21203/rs.3.rs-257837/v1

License: (c) (i) This work is licensed under a Creative Commons Attribution 4.0 International License. Read Full License 


\section{Abstract}

Background: The tumour microenvironment (TME) is closely involved in the genesis and development of gastric carcinoma (GC). However, few studies have investigated the impact of sex on the dynamic modulation of immune and stromal components in the TME.

Methods: In this review, we employed the CIBERSORT and ESTIMATE algorithms to analyse the ratio of tumour-infiltrating immune cells (TICs) and the number of immune and stromal components in 130 female and 218 male GC cases from The Cancer Genome Atlas (TCGA) database. COX regression analysis and a protein-protein interaction (PPI) network were conducted to analyse the differentially expressed genes (DEGs).

Results: The results showed that the Fc fragment of IgG receptor Ila (FCGR2A) in females and GDNF family receptor alpha 1 (GFRA1) in males were predictive factors by intersection analysis of univariate COX and PPI. Moreover, FCGR2A was negatively correlated with the survival of female patients, while GFRA1 was positively related to the survival of male patients. Gene set enrichment analysis (GSEA) demonstrated that genes in the FCGR2A high expression group were primarily enriched in the antigen processing and presentation pathway, while genes in the GFRA1 low expression group were primarily enriched in the cell cycle and DNA replication pathway. Furthermore, CIBERSORT analysis of the proportion of TICs demonstrated that M2 macrophages were positively correlated with FCGR2A expression. B cells, T cells, monocytes and macrophages were positively related to GFRA1 expression.

Conclusions The levels of FCGR2A and GFRA1 might be utilized to outline the prognosis of female and male GC patients, respectively. The results of this study demonstrate the impact of sex on tumour progression and may facilitate the development of therapeutics for GC patients.

\section{Background}

Gastric carcinoma (GC) is reported as the fifth most common cancer and the third leading cause of cancer mortality [1] and is associated with dismal clinical outcomes [2]. The main pathogenesis of GC includes $H$. pylori and Epstein-Barr virus infection, familial inheritance and environmental factors [3-4]. High intake of salts, nitrates and pickled foods and smoking were associated with an increased risk of GC [5-6]. Due to these various causes, GC is a highly heterogeneous disease with diverse molecular signatures and histopathological appearances [7]. Although immunotherapy and neoadjuvant therapy have been widely used in the clinic, the curative effect and 5-year survival rate of GC require further improvement. Tumour microenvironment (TME) formation or transition plays vital roles in GC progression and therapeutic response [8]. The TME is primarily composed of two cell types: stromal components, including fibroblasts, endothelial cells, and mesenchymal stromal cells, and tumour-infiltrating immune cells (TICs), such as macrophages and T cells, whose reaction is regarded as a protective response against tumours [9-10]. TMEs not only secrete many soluble molecules, such as cytokines, chemokines, 
growth factors, and antibodies but also contribute to the presence of hypoxia and acidity, which determine the behaviour of cancers, such as survival, growth, proliferation, and metastasis [11-12].

Previous studies have reported that the regulation of microRNAs and the CXCL1-CXCR2 axis in the TME has great clinicopathological significance for GC [13-14]. However, few studies have investigated the impact of sex on the TME composition and prognosis. Therefore, we screened the transcriptomesequencing results of GC in the TCGA database for further evaluation. The search conditions were as follows: cases (primary sites: stomach; project: TCGA-STAD; disease type: adenomas and adenocarcinomas; gender: male or female); and files (data category: transcriptome profiling; workflow type: HTSeq - FPKM). A total of 130 female cases and 218 male cases were selected for analysis. ESTIMATE and CIBERSORT algorithms were employed to calculate the TIC proportion of stromal and immune components of GC samples. The protein-protein interaction (PPI) network and univariate Cox regression method were performed to analyse differentially expressed genes (DEGs) shared by ImmuneScore and StromalScore. The intersection analysis of core nodes in the PPI network and the top significant factors from Cox regression were employed to identify the key genes. As a result, we identified different predictive biomarkers for the alteration of TME status in female and male GC cases, which were the Fc fragment of IgG receptor Ila (FCGR2A, also known as CD32) and GDNF family receptor alpha 1 (GFRA1), respectively. The subsequent analysis of FCGR2A and GFRA1 included survival and clinicopathological characteristic correlation analysis, gene set enrichment analysis (GSEA), and correlation with TICs. The analysis method followed the study of Ke-Wei Bi et al. [15].

\section{Methods}

\section{Data acquire}

The data of 130 female and 218 male GC cases were obtained from The Cancer Genome Atlas (TCGA) database. The data screening conditions were as follows: Primary site "Stomach"; Program "TCGA"; Project "TCGA-STAD"; Disease type "adenomas and adenocarcinomas"; Data category "transcriptome profiling", Data type "Gene Expression Quantification"; Workflow Type "HTseq-FPKM".

\section{Data analysis}

ImmuneScore, StromalScore and ESTIMATEScore and their correlation with clinicopathological staging characteristics were analysed by using "limma", "estimate", "ggpubr" "survival", and "survminer" packages of R software. GO and KEGG analysis for DEGs were conducted by "clusterProfiler", "org.Hs.eg.db", "enrichplot", and "ggplot2" packages of R software. PPI network and univariate COX analysis were performed by using Cytoscape and "survival" packages of R software. The differentiated expression of FCGR2A and GFRA1 in samples and their correlation with survival and clinicopathological staging characteristics were analysed by "limma" "beeswarm", "ggpubr" "survival", and "survminer" packages of R software. GSEA analysis for samples with high expression and low expression of FCGR2A and GFRA1 were conducted by GSEA software and "plyr", "ggplot2", "grid" and "gridExtra" packages of R software. TIC profile in tumor samples and correlation analysis was conducted by using "CIBERSORT" and "limma" 
packages of R software. Correlation of TICs proportion with FCGR2A and GFRA1 expression were performed by "corrplot", "vioplot”, "ggplot2", and "VennDiagram” packages of R software.

\section{Results}

\section{Correlation of scores with the survival rate}

The correlation between the proportion of immune and stromal cells and the survival rate was evaluated by ImmuneScore and StromalScore, the higher score of which indicated a larger amount of immune or stromal components in the TME. ESTIMATEScore denotes the comprehensive proportion of immune and stromal cells. The results showed that in female samples, the StromalScore and ESTIMATEScore had evident positive correlations with the survival rate, while the ImmuneScore was the opposite (Fig. 1A-C). Meanwhile, all three scores had a notably positive relationship with the survival rate in male samples (Fig. 1D-F). The results indicated that both the immune and stromal components are important for the prognosis of GC patients.

\section{Correlation Of Scores With Clinicopathological Staging Characteristics}

To determine the influence of the immune and stromal components on the clinicopathological staging characteristics, we evaluated the correlation of the ImmuneScore, StromalScore and ESTIMATEScore with tumour stage, grade, $\mathrm{T}$ classification, $\mathrm{M}$ classification and $\mathrm{N}$ classification. In female samples, the ImmuneScore, StromalScore and ESTIMATEScore had significant positive correlations with tumour grade, especially between grade II and grade III (Fig. 2). In contrast, other clinicopathological staging characteristics showed no evident relationship (Fig. 2). In male samples, the ImmuneScore, StromalScore and ESTIMATEScore exhibited significant correlations with tumour grade, as well as T classification (Fig. 3). In addition, the ESTIMATEScore had an evident relationship with tumour stage. The results implied that the immune and stromal components were related to GC development.

DEGs Shared by ImmuneScore and StromalScore Were Predominantly Enriched for Immune-Related Genes

DGEs were identified by comparison analysis between high- and low-ImmuneScore/StomalScore samples and used for further GO and KEGG analysis. In female samples, a total of 811 DEGs were obtained, among which 741 were upregulated and 70 were downregulated (Fig. 4A-D). In male samples, a total of 513 DEGs were obtained, among which 439 were upregulated and 74 were downregulated (Fig. 5A-D). These DEGs were potential determining factors for TME status.

Gene ontology (GO) enrichment analysis showed that the main functions of DEGs in the female sample were cell-cell adhesion and T cell activation (Fig. 4E), and Kyoto Encyclopedia of Genes and Genomes (KEGG) enrichment analysis also showed enrichment of the cell adhesion pathway and chemokine 
signalling pathway (Fig. 4F). Meanwhile, GO results suggested that DEGs in male samples oversaw immune cell proliferation and migration (Fig. 5E). KEGG analysis showed the enrichment of chemokine signalling pathways and chemokine-chemokine receptor interaction pathways. Therefore, the primary functions of DEGs in females and males seemed to map to immune- and chemokine-related activities.

\section{Intersection Analysis of the PPI Network and Univariate Cox Regression}

The STRING database and Cytoscape software were applied to construct a PPI network of 811 DEGs in female samples, as well as 513 DEGs in male samples (Fig. 6A, E), and the top 30 genes ordered by the number of adjacent nodes are shown in Fig. 6B and $\mathrm{F}$. Meanwhile, univariate Cox regression analysis was performed to evaluate the significant factors for the survival of GC patients in females and males (Fig. 6C and G). Subsequently, intersection analysis between the top leading genes of the PPI network and Cox regression was performed to screen the key mediators of prognosis. The results showed that the overlapping genes from the above analyses were FCGR2A in female samples and GFRA1 in male samples (Fig. 6D and H).

\section{Correlation of FCGR2A and GFRA1 Expression with Survival and Clinicopathological Staging Characteristics in GC Patients}

All female samples were grouped into FCGR2A high- and low-expression groups compared with FCGR2A median expression; similarly, all male samples were grouped into GFRA1 high- and low-expression groups. FCGR2A was evidently highly expressed in female GC samples compared to normal samples (Fig. 7A), and the same result was obtained in pairing analysis between the GC samples and normal samples derived from the same patient (Fig. 7B). GC female patients with high expression of FCGR2A had a significantly lower survival rate than those with low expression of FCGR2A (Fig. 7C). Additionally, FCGR2A was highly expressed in G2 grade compared with G3 grade (Fig. 7D). For other clinicopathological staging characteristics, there were no significant changes (Fig. 7E-H). Whereas GFRA1 was significantly downregulated in male GC samples compared to normal samples (Fig. 7l), the same result was obtained in pairing analysis between the GC samples and normal samples derived from the same patient (Fig. 7J). Male GC patients with low GFRA1 expression had a significantly lower survival rate than those with high GFRA1 expression (Fig. 7K). GFRA1 expression in stage II and stage III had significant changes compared to stage I. Additionally, GFRA1 expression in T3 and T4 had significant changes compared to T1 phase (Fig. 7M-N). For other clinicopathological staging characteristics, there were no significant changes (Fig. 7L, O-P). The above results showed that the expression of FCGR2A in the TME had a positive correlation with the prognosis of female GC patients, while GFRA1 in the TME had a negative correlation with the prognosis of male GC patients.

\section{Gsea Of Fcgr2a And Gfra1}

GSEA was performed to analyse the high- and low-expression groups compared with the median levels of FCGR2A and GFRA1 expression, respectively. The genes in the FCGR2A high expression group were 
mainly enriched in cell adhesion and chemokine signalling pathways (Fig. 8A), while the genes in the FCGR2A low expression group were mainly enriched in metabolic pathways (Fig. 8B). The genes in the GFRA1 high expression pathway were mainly enriched in the calcium signalling pathway and cell adhesion pathway (Fig. 8C). For the GFRA1 low-expression group, the genes were enriched in the cell cycle and DNA replication pathways (Fig. 8D). These results indicated that FCGR2A and GFRA1 might be potential indicators for the status of the TME in females and males, respectively.

\section{Correlation of FCGR2A and GFRA1 with the proportion of TICs}

To further determine the correlation between FCGR2A expression and the immune environment, the rate of tumour infiltrating immune cell subsets was analysed using the CIBERSORT algorithm. Twenty-two kinds of immune cells in female samples and 21 kinds of immune cells in male samples were obtained (Fig. 9A and B). The correlation between TICs in female and male samples is shown in Fig. 9C and D. Next, difference and correlation analyses were conducted, the results of which showed that four kinds of TICs were correlated with the expression of FGCR2A. Among these TICs, memory B cells, regulatory T cells and activated dendritic cells were negatively correlated with FCGR2A expression, whereas only M2 macrophages were positively correlated with FCGR2A expression (Fig. 10A-C). Meanwhile, 10 kinds of TICs were correlated with the expression of GFRA1. Among them, naïve B cells, memory B cells, plasma cells, resting CD4 memory T cells, regulatory $T$ cells (Tregs), monocytes and resting mast cells were positively correlated with GFRA1 expression. Four kinds of TICs were negatively correlated with GFRA1 expression, including activated memory CD4 T cells, follicular helper T cells, M0 macrophages and M1 macrophages (Fig. 11A-C). These results further proved that FCGR2A and GFRA1 affected the immune activity of the female and male TMEs, respectively.

\section{Discussion And Conclusions}

In this data mining study, we attempted to determine TME-related genes that play critical roles in survival, as well as clinicopathological staging characteristics in GC patients from the TCGA database. The important role of TME had long been studied. Chunwei Peng et al. gathered data from 494 GC patients for TME analysis, the results of which demonstrated that the tumour-stromal ratio could be easily implemented in routine pathology diagnostics [16]. Xiaolong Wu reported that GC patients with high levels of T cell inflammation were more likely to benefit from adjuvant chemotherapy [17]. In contrast, it was reported that stromal-relevant genes were identified as adverse prognostic factors in GC [18]. Interestingly, our study found that a high StromalScore was positively correlated with the survival rate of both female and male patients, while a high ImmuneScore was negatively correlated with the survival rate in female samples and positively correlated with male samples. Further analysis screened FCGR2A and GFRA 1 as significant factors in immune activates in female and male GC patients, respectively. More importantly, these genes were identified as indicators for the status of the TME through a series of bioinformatics analyses. 
FCGR2A encodes one member of a family of immunoglobulin Fc receptor genes found on the surface of phagocytosis cells, including macrophages and neutrophils [19]. It had two polymorphic alleles at the amino acid position, which was associated with an increased risk of GC patients [20]. Clinical studies have reported that FCGR2A polymorphisms are associated with the clinical outcome of tumours, including colorectal cancer, neuroblastoma and renal cell carcinoma [21-24]. In contrast, either FCGR2A or FCGR3A polymorphisms affect the clinical outcome of follicular lymphoma patients [25]. In addition, it was reported that the FCGR2A variant rs1801274 was evidently correlated with gastric cancer risk [26]. Consistently, our analysis found that FCGR2A was highly expressed in female GC patients compared to healthy people and negatively associated with the survival rate. However, only one kind of TIC was positively correlated with the expression of FCGR2A, which is M2 macrophages. As the main tumourinfiltrating leukocytes, macrophages play vital roles in cancer-related inflammation [27]. Depending on the polarization status, tumour-associated macrophages (TAMs) can either promote antitumour immune responses (M2) or contribute to tumour progression (M1) [28]. The ratio of the M2/M1 phenotype is relevant in tumour initiation, progression, and dissemination [29]. FCGR2A phosphorylation is important for activation of macrophage phagocytosis [30], as well as mediating the production of proinflammatory cytokines [31]. Therefore, highly expressed FCGR2A in macrophages promotes the M2-M1 switch by inducing proinflammatory cytokine production, which has a negative influence on the survival rate.

As the receptor of glial cell-derived neurotrophic factor (GDNF), GFRA1 is involved in the regulation of proliferation, differentiation, and migration of neuronal cells [32]. Additionally, GFRA1 is upregulated in many cancers and participates in cancer cell progression, metastasis, and autophagy by activating the classic RET-RAS-ERK, RET-RAS-PI3K-AKT and SRC-AMPK signalling pathways [33-36]. In contrast to previous studies, our analysis showed that the expression of GFRA1 was significantly decreased in male GC patients and positively correlated with the survival rate. The underlying mechanism warrants further investigation. Based on TIC analysis, 10 kinds of cells were correlated with GFRA1 expression, mainly including B cells and T cells. Our study also implied that low expression of GFRA1 facilitates the cell cycle and DNA replication pathway in male GC patients based on GSEA.

The present review provided new insight into susceptibility factors of immunoregulatory gene variants in the carcinogenesis of GC in both sexes. We determined TME-related genes in both female and male GC samples through functional enrichment analysis in the TCGA database. FCGR2A might be a negative indicator for the survival of female patients, while GFRA1 serves as a positive indicator for the survival of male patients. Therefore, further investigation should be conducted to clarify the accurate function and potential role of FCGR2A and GFRA1 in GC patients.

\section{Abbreviations}

TME: Tumor microenvironment; GC: gastric carcinoma; TIC: tumor-infiltrating immune cell; TCGA: The Cancer Genome Atlas; PPI: protein-protein interaction; DEGs: differentially expressed genes; FCGR2A: Fc fragment of IgG receptor Ila; GFRA1: GDNF family receptor alpha 1; GSEA: Gene Set Enrichment Analysis; Tregs: T cells regulatory; TAMs: tumor-associated macrophages. 


\section{Declarations}

\section{Ethics approval and consent to participate}

Not applicable.

\section{Consent for publication}

Not applicable.

\section{Availability of data and material}

Datasets generated and analyzed during the current study are available from

The Cancer Genome Atlas, https://portal.gdc.cancer.gov.

\section{Competing interests}

The authors declare that there is no conflict of interests in this study.

\section{Funding}

This work was supported by the National Nature Science Foundation of China (NO.81473793, 81603711). Tianjin Education Commission Research Project (NO.20120210). The funding bodies had no role in the study design, the collection, analysis, and interpretation of data, or the writing of the article and the decision to submit it for publication.

\section{Authors' contributions}

$\mathrm{XH}$ wrote the paper. FL, LJ, XW, YP, HL, WZ, XL, QS performed the collected the data. All authors read and approved the final manuscript.

\section{Acknowledgements}

Not applicable.

\section{References}

1. Torre LA, Bray F, Siegel RL, Ferlay J, Lortet-Tieulent J, Jemal A. Global cancer statistics 2012. CA Cancer J Clin. 2015;65:87-108.

2. Waldum HL, Fossmark R. Types of Gastric Carcinomas. Int J Mol Sci. 2018;19:4109.

3. Eric VC, Sagaert X, Topal B, Haustermans K, Prenen H. Gastric cancer. The Lancet. 2016;388:265464.

4. Oliveira C, Pinheiro H, Figueiredo J, Seruca R, Carneiro F. Familial gastric cancer: genetic susceptibility, pathology, and implications for management. Lancet Oncol. 2015;16:e60-70. 
5. Poorolajal J, Moradi L, Mohammadi Y, Cheraghi Z, Gohari-Ensaf F, Cheraghi Z, Gohari-Ensaf F. Risk factors for stomach cancer: a systematic review and meta-analysis. Epidemiol Health. 2020;42:e2020004.

6. Ladeiras LR, Pereira A, Nogueira A, et al. Smoking and gastric cancer: systematic review and metaanalysis of cohort studies. Cancer Causes Control. 2008;19:689-701.

7. Nanki K, Toshimitsu K, Takano A, Fujii M, Shimokawa M, Ohta Y, et al. Divergent Routes toward Wnt and R-spondin Niche Independency during Human Gastric Carcinogenesis. Cell. 2018;174:856-69.

8. Son B, Lee S, Youn H, Kim E, Kim W, Youn B. The role of tumor microenvironment in therapeutic resistance. Oncotarget. 2017;8:3933-45.

9. Ma B, Wells A, Clark AM. The pan-therapeutic resistance of disseminated tumor cells: Role of phenotypic plasticity and the metastatic microenvironment. Semin Cancer Biol. 2019;60:138-47.

10. Zhi HZ, Cheng DJ, Jiang Z, et al. The prognostic value and pathobiological significance of Glasgow microenvironment score in gastric cancer. J Cancer Res Clin Oncol. 2017;143:883-94.

11. Hye WC, Jong BL. Role of the tumor microenvironment in the pathogenesis of gastric carcinoma. World J Gastroenterol. 2014;20:1667-80.

12. Liu H, Ni S, Wang H, Zhang Q, Weng W. Charactering tumor microenvironment reveals stromal-related transcription factors promote tumor carcinogenesis in gastric cancer. Cancer Med. 2020;9:5247-57.

13. Zhang H, Bai M, Deng T, Liu R, Wang X, Qu Y, et al. Cell-derived microvesicles mediate the delivery of miR-29a/c to suppress angiogenesis in gastric carcinoma. Cancer Lett. 2016;375:331-9.

14. Kasashima H, Yashiro M, Nakamae H, Masuda G, Kinoshita H, Morisaki T, et al. Clinicopathologic significance of the CXCL1-CXCR2 axis in the tumor microenvironment of gastric carcinoma. PLOS ONE. 2017;12:e0178635.

15. Ke WB, Xu GW, Xiao XQ, Bo L. BTK Has Potential to Be a Prognostic Factor for Lung Adenocarcinoma and an Indicator for Tumor Microenvironment Remodeling: A Study Based on TCGA Data Mining. Front Oncol. 2020;10:424.

16. Peng CW, Liu JY, Yang GF, Li Y. The tumor-stromal ratio as a strong prognosticator for advanced gastric cancer patients: proposal of a new TSNM staging system. J Gastroenterol. 2018;53:606-17.

17. Wu X, Gao X, Xing X, Wen X, Li Z, Ji J. The T-Cell-Inflammation status can predict outcomes of adjuvant chemotherapy in patients with gastric cancer. Ann Surg Oncol. 2020. doi:10.1245/s10434020-09005-4.

18. Wei S, Lu J, Lou J, Shi C, Mo S, Shao Y, et al. Gastric cancer tumor microenvironment characterization reveals stromal-related gene signatures associated with macrophage infiltration. Front Genet. 2020;11:663.

19. Treffers LW, Houdt MV, Bruggeman CW, Heineke MH, Zhao XW, et al. FcyRIIlb Restricts AntibodyDependent Destruction of Cancer Cells by Human Neutrophils. Front Immunol. 2019;9:3124.

20. Viansone AA, Boggiani D, Musolino A. Prognostic Role of Immunoglobulin G Fragment C Receptor Polymorphisms in Solid Tumors. JAMA Oncol. 2018;4:132-3. 
21. Cheung NK, Sowers R, Vickers AJ, Cheung IY, Kushner BH, Gorlick R. FCGR2APolymorphism Is Correlated With Clinical Outcome After Immunotherapy of Neuroblastoma With Anti-GD2 Antibody and Granulocyte Macrophage Colony-Stimulating Factor. J Clin Oncol. 2006;24:2885-90.

22. Zhang W, Gordon M, Schultheis AM, Yang DY, Nagashima F, Azuma M, et al. FCGR2A and FCGR3A Polymorphisms Associated With Clinical Outcome of Epidermal Growth Factor Receptor-Expressing Metastatic Colorectal Cancer Patients Treated With Single-Agent Cetuximab. J Clin Oncol. 2007;25:3712-8.

23. Gavin PG, Song N, Kim SR, Lipchik C, Johnson NL. Association of Polymorphisms in FCGR2A and FCGR3A With Degree of Trastuzumab Benefit in the Adjuvant Treatment of ERBB2/HER2-Positive Breast Cancer Analysis of the NSABPB-31Trial. JAMA Oncol. 2017;3:335-41.

24. Erbe AK, Wang W, Goldberg J, Gallenberger M, Kim K, Carmichael L, et al. FCGR Polymorphisms Influence Response to IL2 in Metastatic Renal Cell Carcinoma. Clin Cancer Res. 2017;23:2159-68.

25. Ghesquières $\mathrm{H}$, Cartron $\mathrm{G}$, Seymour JF, et al. Clinical outcome of patients with follicular lymphoma receiving chemoimmunotherapy in the PRIMA study is not affected by FCGR3A and FCGR2A polymorphisms. Blood. 2012;120:2650-7.

26. Xia HZ, Du WD, Wu Q, Chen G, Zhou Y, Tang XF, et al. E-selectin rs5361 and FCGR2A rs1801274 variants were associated with increased risk of gastric cancer in a Chinese population. Mol Carcinog. 2012;51:597-607.

27. Mantovani A, Sozzani S, Locati M, Allavena P, Sica A. Macrophage polarization: tumor-associated macrophages as a paradigm for polarized M2 mononuclear phagocytes. Trends Immunol. 2002;23:549-55.

28. Biswas SK, Mantovani A. Macrophage plasticity and interaction with lymphocyte subsets: cancer as a paradigm. Nat Immunol. 2010;11:889-96.

29. Dan H, Liu S, Liu J, Liu D, Yin F, Wei Z, et al. RACK1 promotes cancer progression by increasing the M2/M1 macrophage ratio via the NF-KB pathway in oral squamous cell carcinoma. Mol Oncol. 2020;14:795-807.

30. Vogelpoel LT, Hansen IS, Rispens T, Muller FJ, van Capel TM, Turina MC, et al. Fc gamma receptorTLR cross-talk elicits pro-inflammatory cytokine production by human M2 macrophages. Nat Commun. 2014;5:5444.

31. Peluso MO, Adam A, Armet CM, Zhang L, O'Connor RW, Lee BH, et al. The Fully human anti-CD47 antibody SRF231 exerts dual-mechanism antitumor activity via engagement of the activating receptor CD32a. J Immunother Cancer. 2020;8:e000413.

32. Ibanez CF, Andressoo JO. Biology of GDNF and its receptors-Relevance for disorders of the central nervous system. Neurobiol Dis. 2017;97:80-9.

33. Mihwa K, Dae JK. GFRA1: A Novel Molecular Target for the Prevention of Osteosarcoma Chemoresistance. Int J Mol Sci. 2018;19:1078.

34. Kim M, Jung JY, Choi S, et al. GFRA1 promotes cisplatin-induced chemoresistance in osteosarcoma by inducing autophagy. Autophagy. 2017;13:149-68. 
35. Picco G, Julien S, Brockhausen I, et al. Over-expression of ST3Gal-I promotes mammary tumorigenesis. Glycobiology. 2010;20:1241-50.

36. Ma WR, Xu P, Liu ZJ, Zhou J, et al. GFRA1 Impact of gene reactivation by DNA demethylation on prognosis of patients with metastatic colon cancer. World J Gastroenterol. 2020;26:184-98.

\section{Figures}
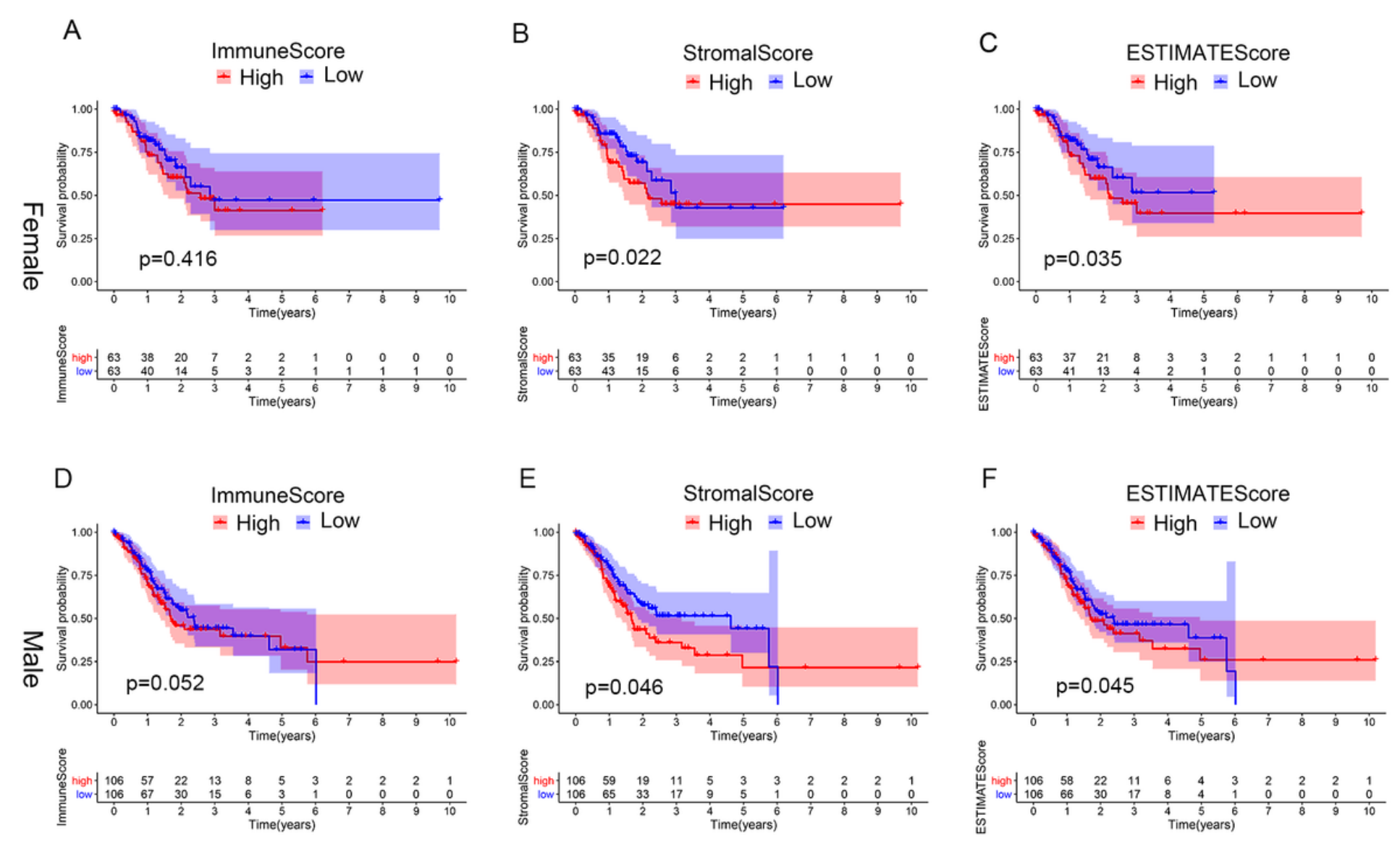

\section{Figure 1}

Correlation of scores with the survival of female and male GC patients. (A) Kaplan-Meier survival analysis for female GC patients grouped into high or low scores in ImmuneScore (A), StromalScore (B) and ESTIMATEScore (C) determined by the comparison with the median; Kaplan-Meier survival analysis for male GC patients grouped into high or low scores in ImmuneScore (D), StromalScore (E) and ESTIMATEScore $(F)$ determined by the comparison with the median. 

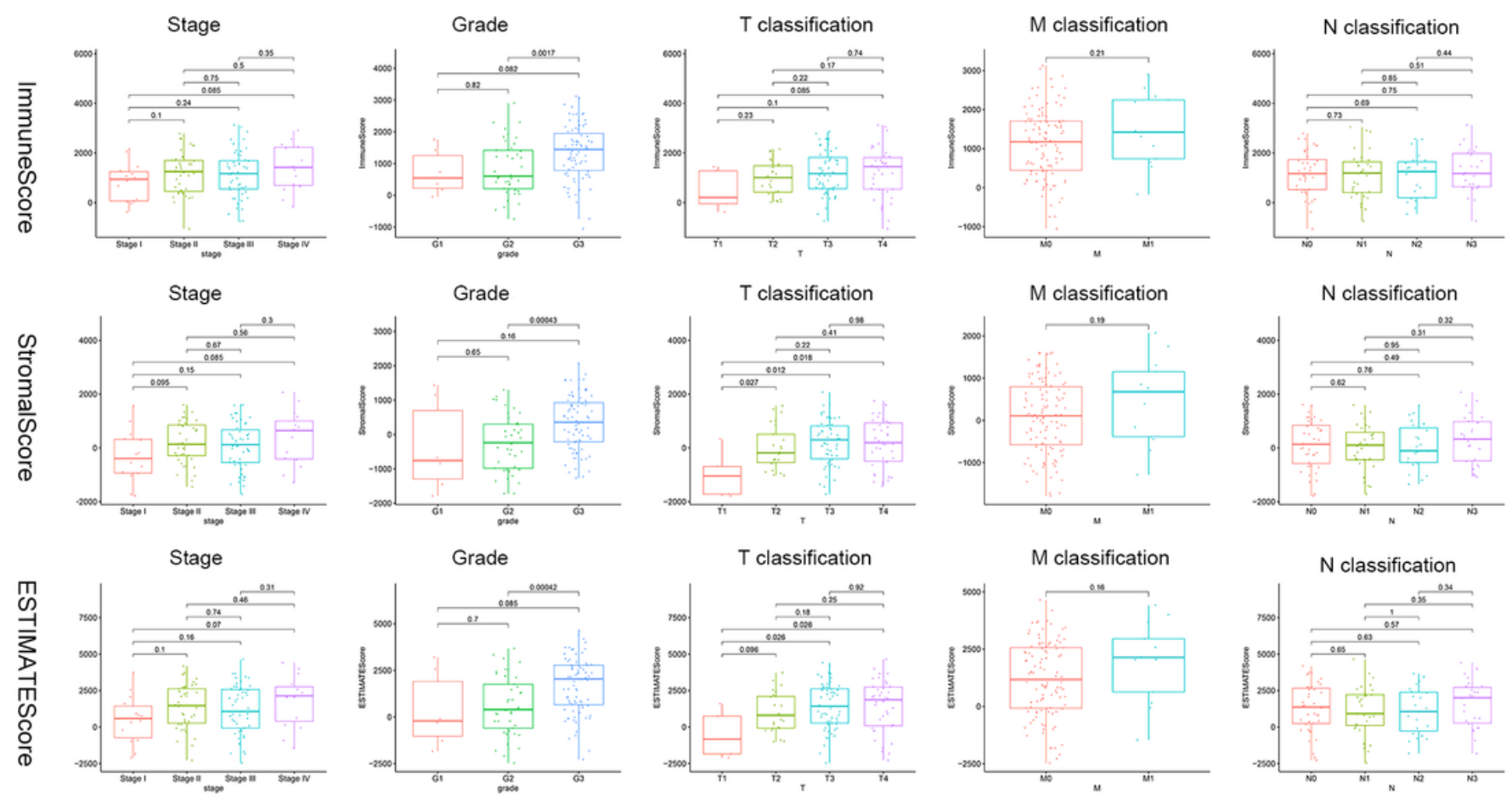

\section{Figure 2}

Correlation of ImmuneScore, StromalScore and ESTIMATEScore with clinicopathological staging characteristics in female GC patients. (A) ImmuneScore of stage, grade, T classification, $M$ classification and $\mathrm{N}$ classification. (B) Stromal score of stage, grade, $\mathrm{T}$ classification, $\mathrm{M}$ classification and $\mathrm{N}$ classification. (C) ESTIMATEScore of stage, grade, T classification, $\mathrm{M}$ classification and $\mathrm{N}$ classification. The statistical method was the Kruskal-Wallis rank sum test. 

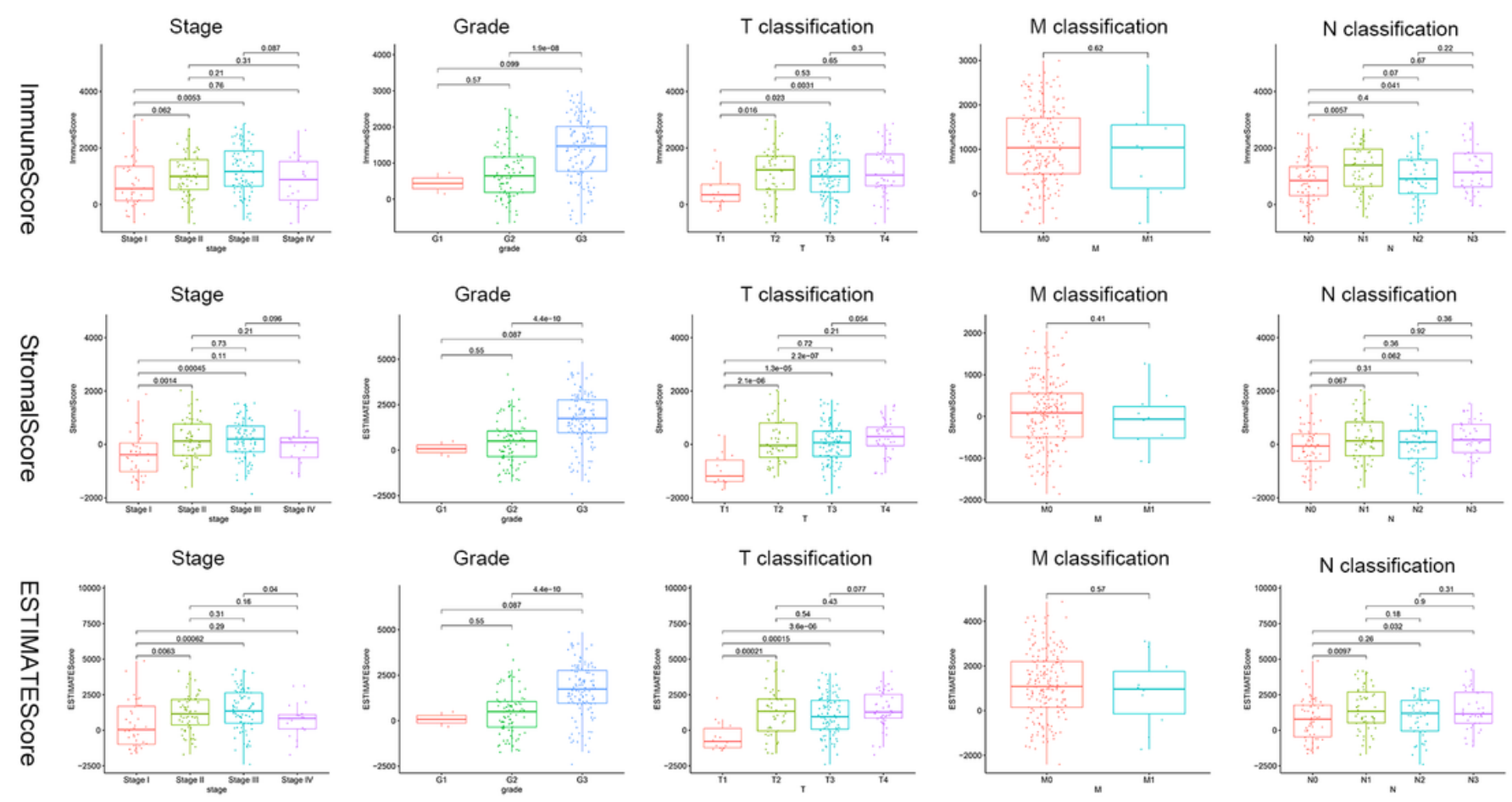

\section{Figure 3}

Correlation of ImmuneScore, StromalScore and ESTIMATEScore with clinicopathological staging characteristics in male GC patients. (A) ImmuneScore, StromalScore (B) and ESTIMATEScore (C) of stage, grade, $\mathrm{T}$ classification, $\mathrm{M}$ classification and $\mathrm{N}$ classification. The statistical method was the Kruskal-Wallis rank sum test. 
A

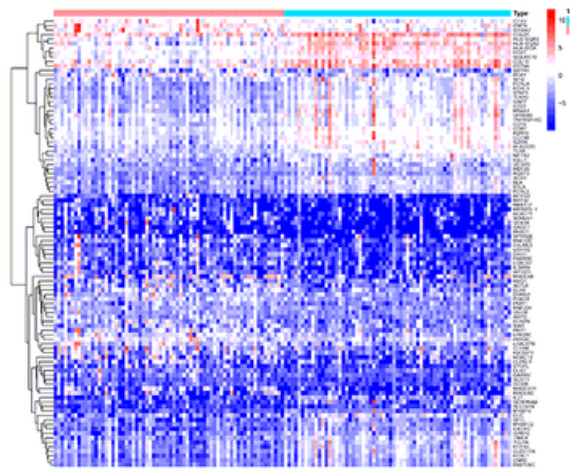

B

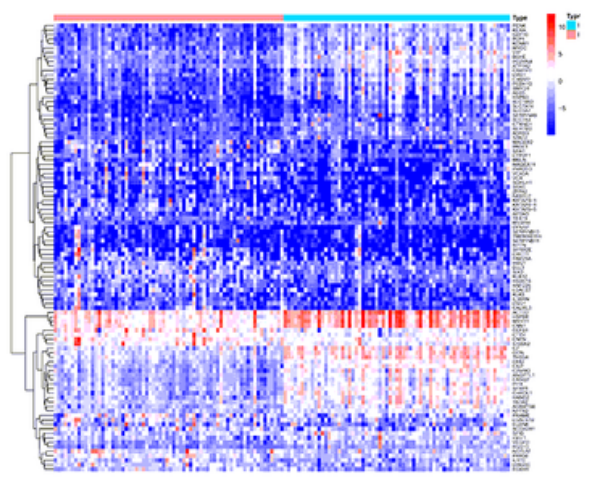

C

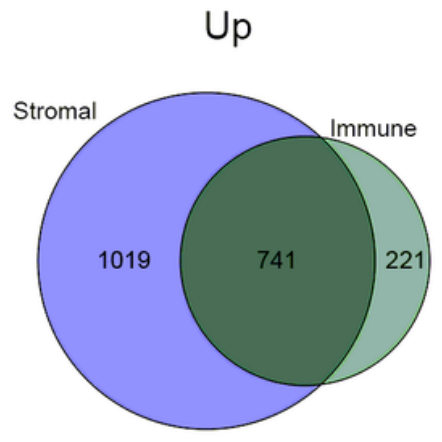

D

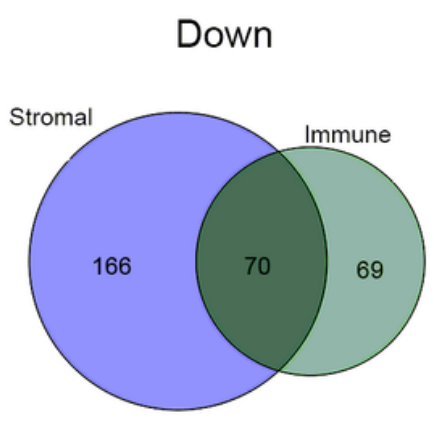

$\mathrm{E}$

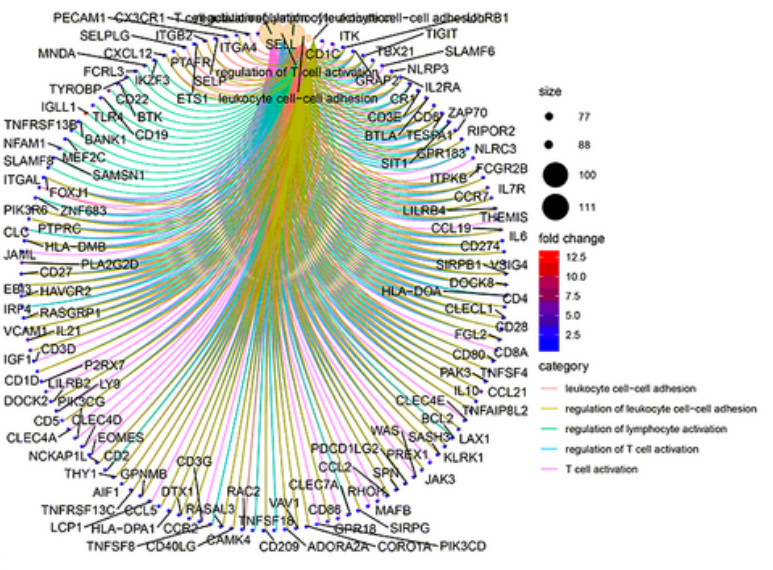

$\mathrm{F}$

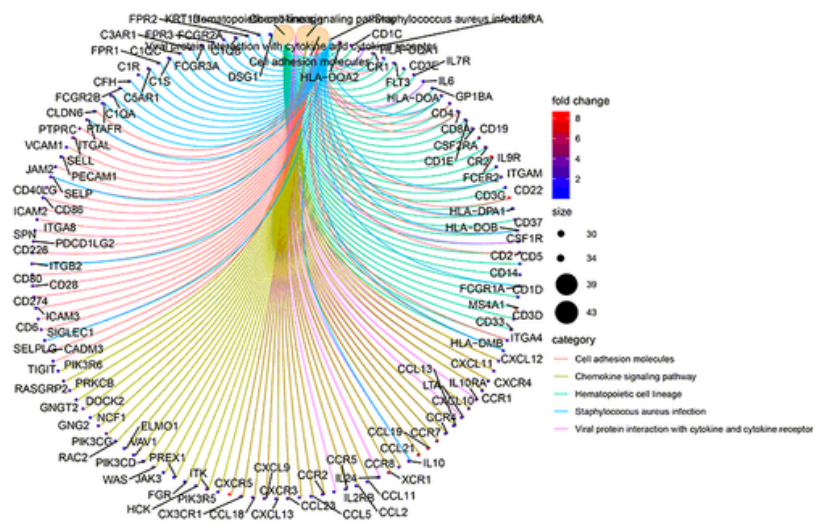

\section{Figure 4}

GO and KEGG analysis for DEGs in female GC patients. Heatmap for DEGs generated by comparison of the high scores and the low score group in ImmuneScore (A) and StromalScore (B). DEGs were determined by the Wilcoxon rank sum test with $\mathrm{q}<0.05$ and fold-change $>1$ after log2 transformation as the significance threshold. Venn plots of upregulated (C) and downregulated (D) DEGs shared by ImmuneScore and StromalScore. GO (E) and KEGG (F) enrichment analysis for 811 DEGs. P-values $<0.05$ were determined to indicate significant enrichment. 

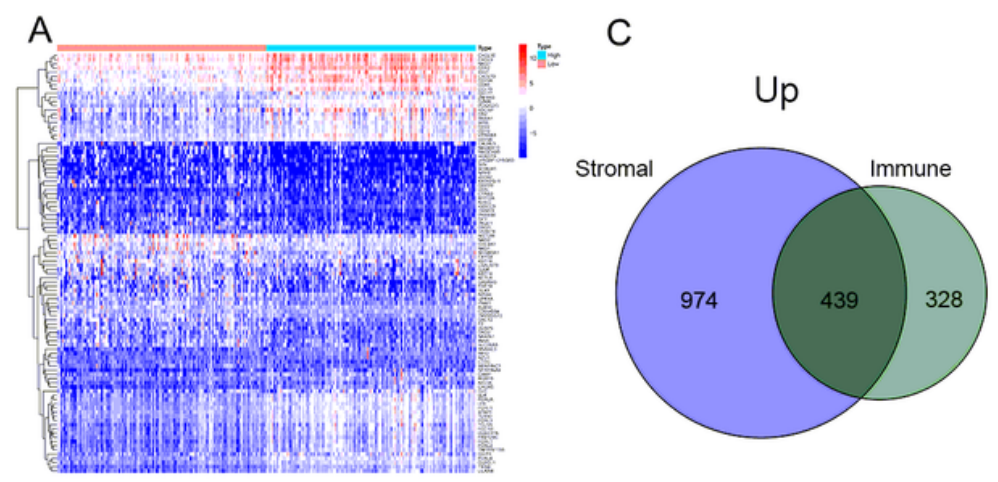

E
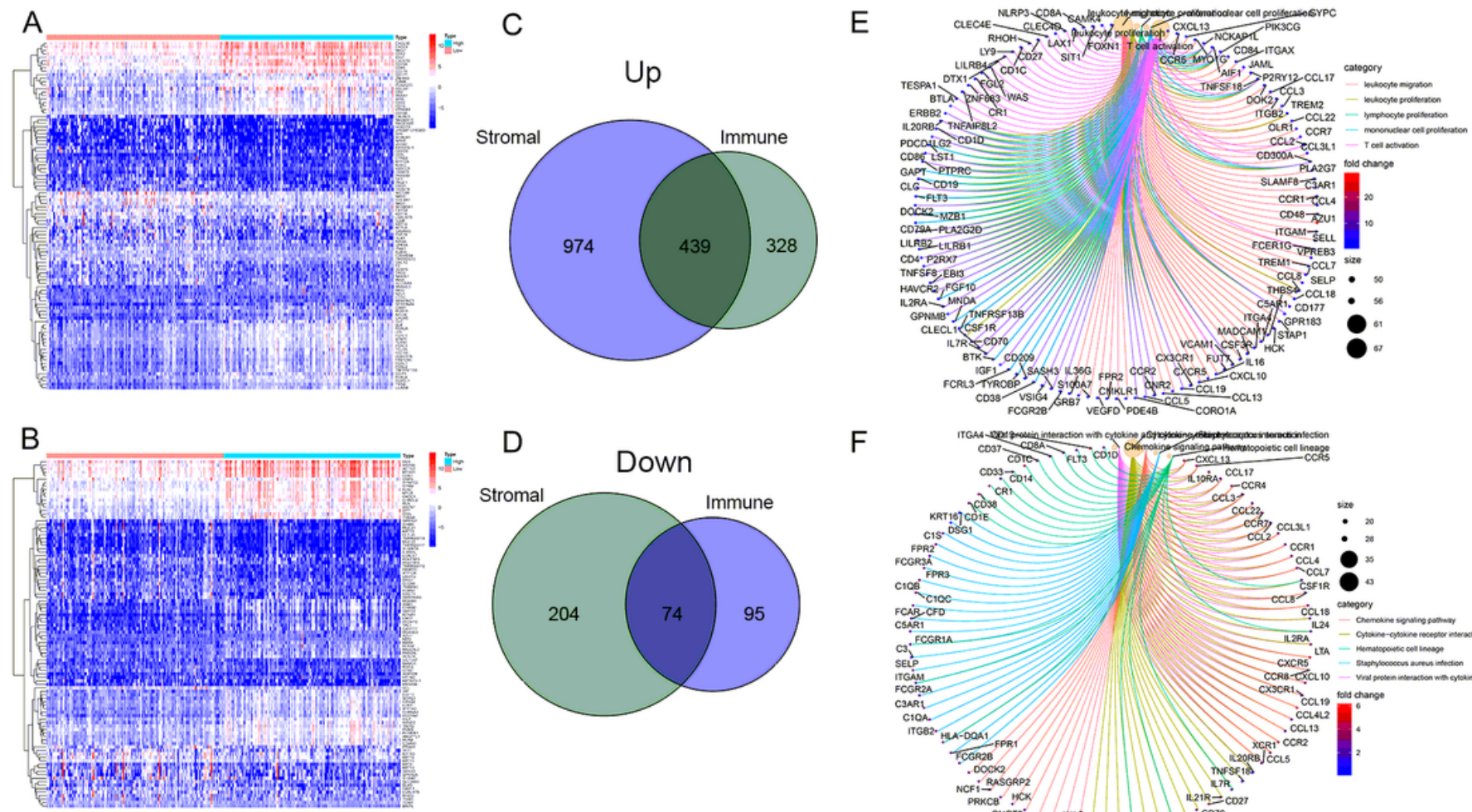

F

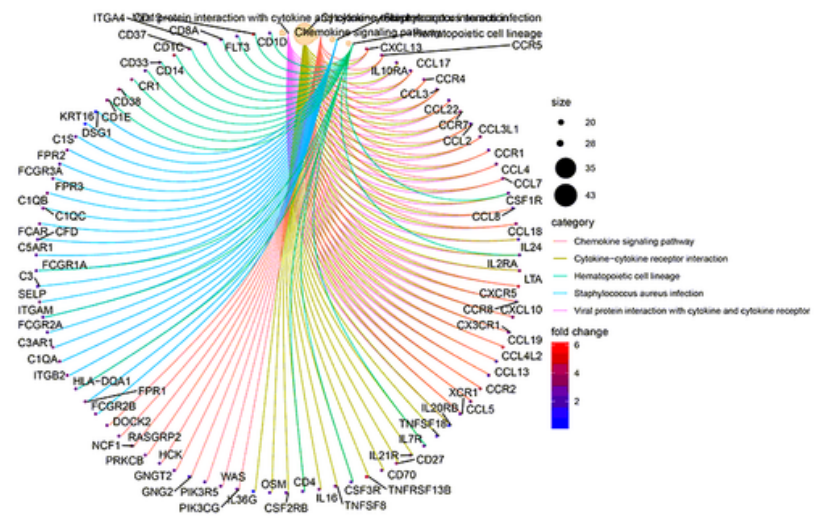

Figure 5

GO and KEGG analysis for DEGs in male GC patients. Heatmap for DEGs generated by comparison of the high scores and the low score group in ImmuneScore (A) and StromalScore (B). DEGs were determined by the Wilcoxon rank sum test with $\mathrm{q}<0.05$ and fold-change $>1$ after log2 transformation as the significance threshold. Venn plots of upregulated (C) and downregulated (D) DEGs shared by ImmuneScore and StromalScore. GO (E) and KEGG (F) enrichment analysis for 513 DEGs. P-values $<0.05$ were determined to indicate significant enrichment. 
A

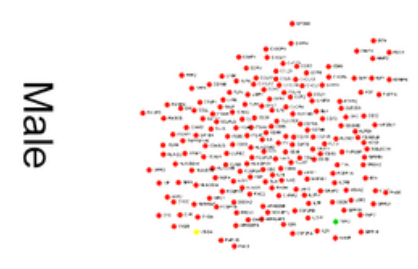

E

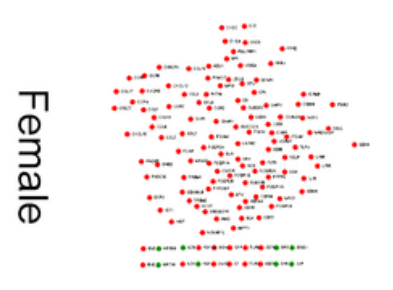

B

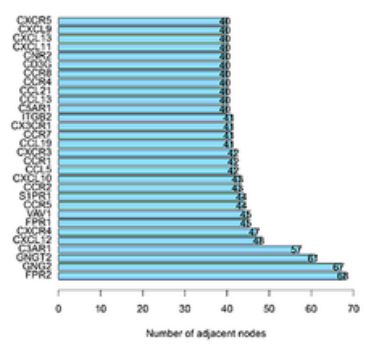

$\mathrm{F}$

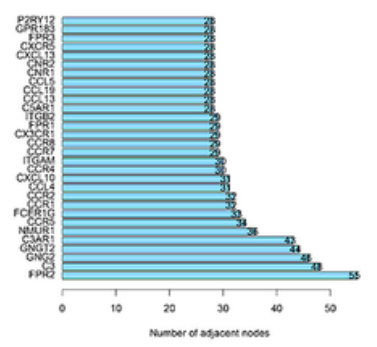

C

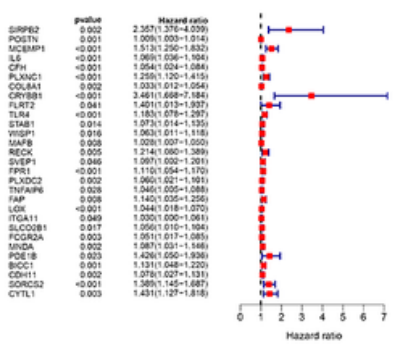

G

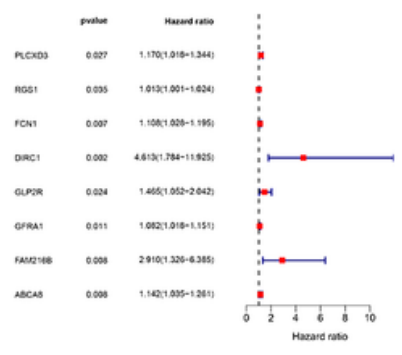

Intersectoin
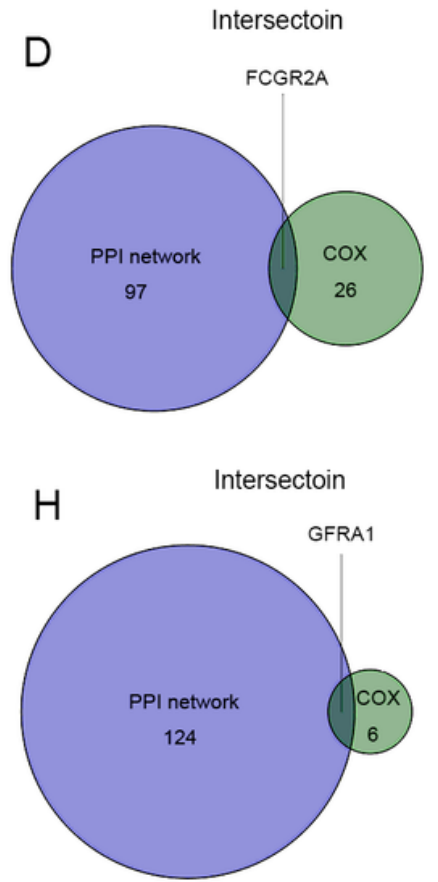

\section{Figure 6}

Protein-protein interaction network and univariate Cox analysis in female and male GC patients.

Interaction network constructed with the nodes with interaction confidence value $>0.9$. in female $(A)$ and male $(E)$. The top 30 genes ordered by the number of nodes in females $(B)$ and males $(F)$. Univariate Cox regression analysis with 811 DEGs in females (C) and 513 DEGs (G) in males, listing the top significant factors with $p<0.005$. Venn plot showing the common factors shared by leading 97 and 125 nodes in PPI and top significant factors in univariate Cox in females (D) and males (H), respectively. 
A

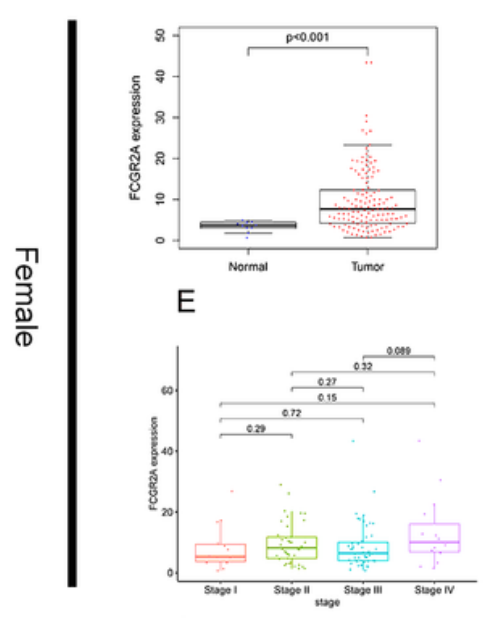

।

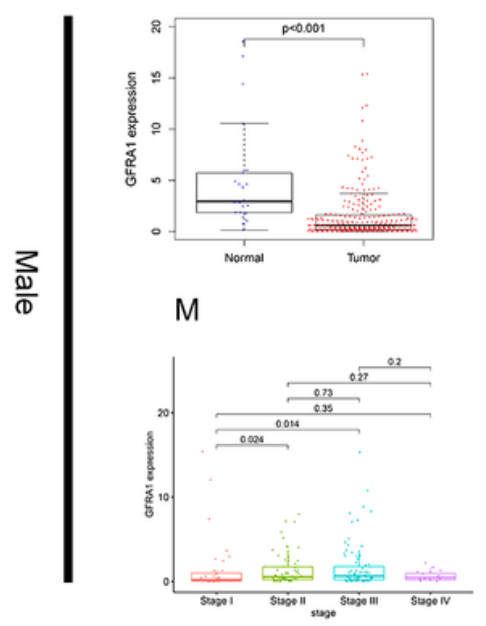

B

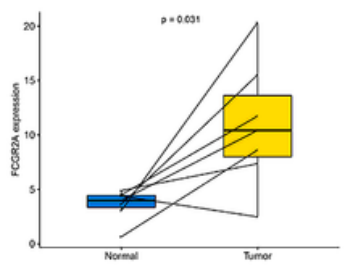

$\mathrm{F}$

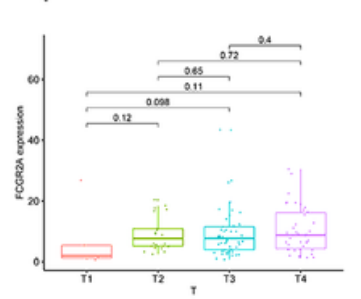

$J$

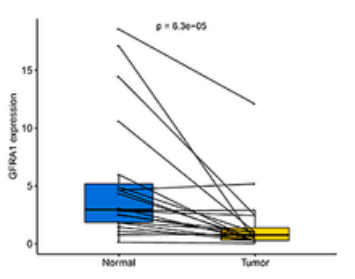

$\mathrm{N}$

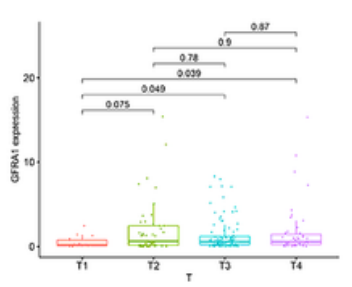

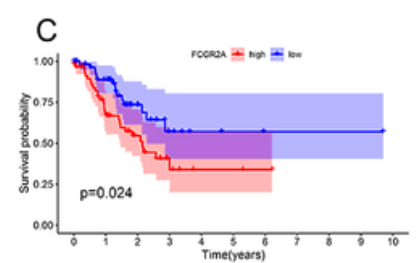

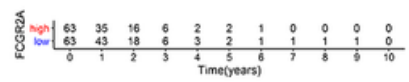

G
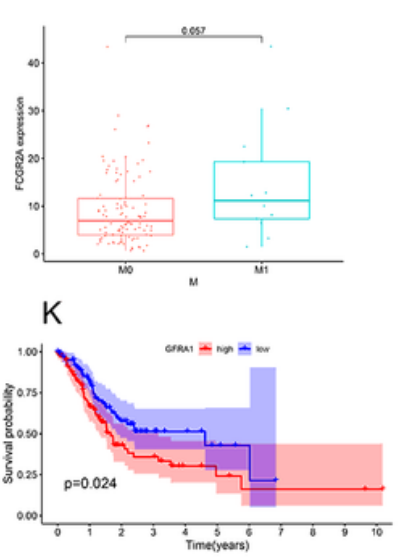

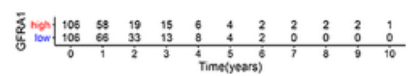

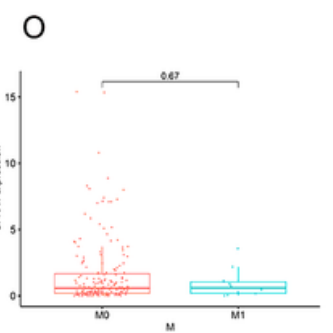

D
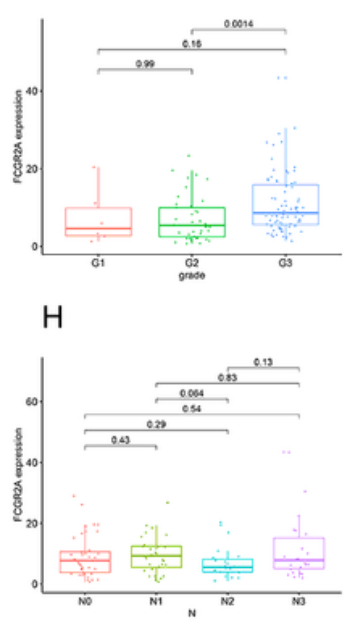

L
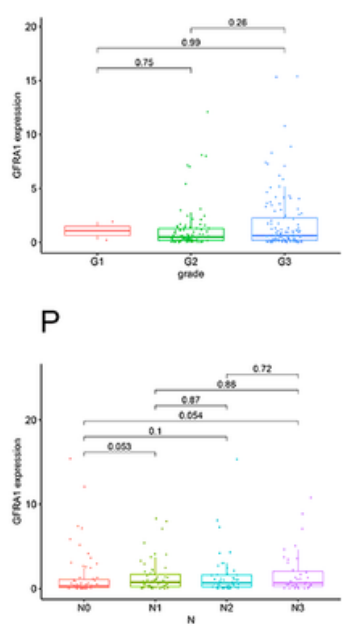

\section{Figure 7}

Differential expression of FCGR2A and GFRA1 in samples and correlation with survival and clinicopathological staging characteristics of female and male GC patients, respectively. Differentiated expression of FCGR2A (A) and GFRA1 (I) in normal and tumour samples. Paired differentiation analysis for the expression of FCGR2A (B) and GFRA1 (J) in normal and tumour samples derived from the same patient. Survival analysis for GC patients with different FCGR2A (C) and GFRA1 (K) expression levels. Patients were labelled with high expression or low expression depending on the comparison with the median expression level. The correlation of FCGR2A (D-H) and GFRA1 (L-P) expression with clinicopathological staging characteristics. The Wilcoxon rank sum or Kruskal-Wallis rank sum test served as the statistical significance test. 

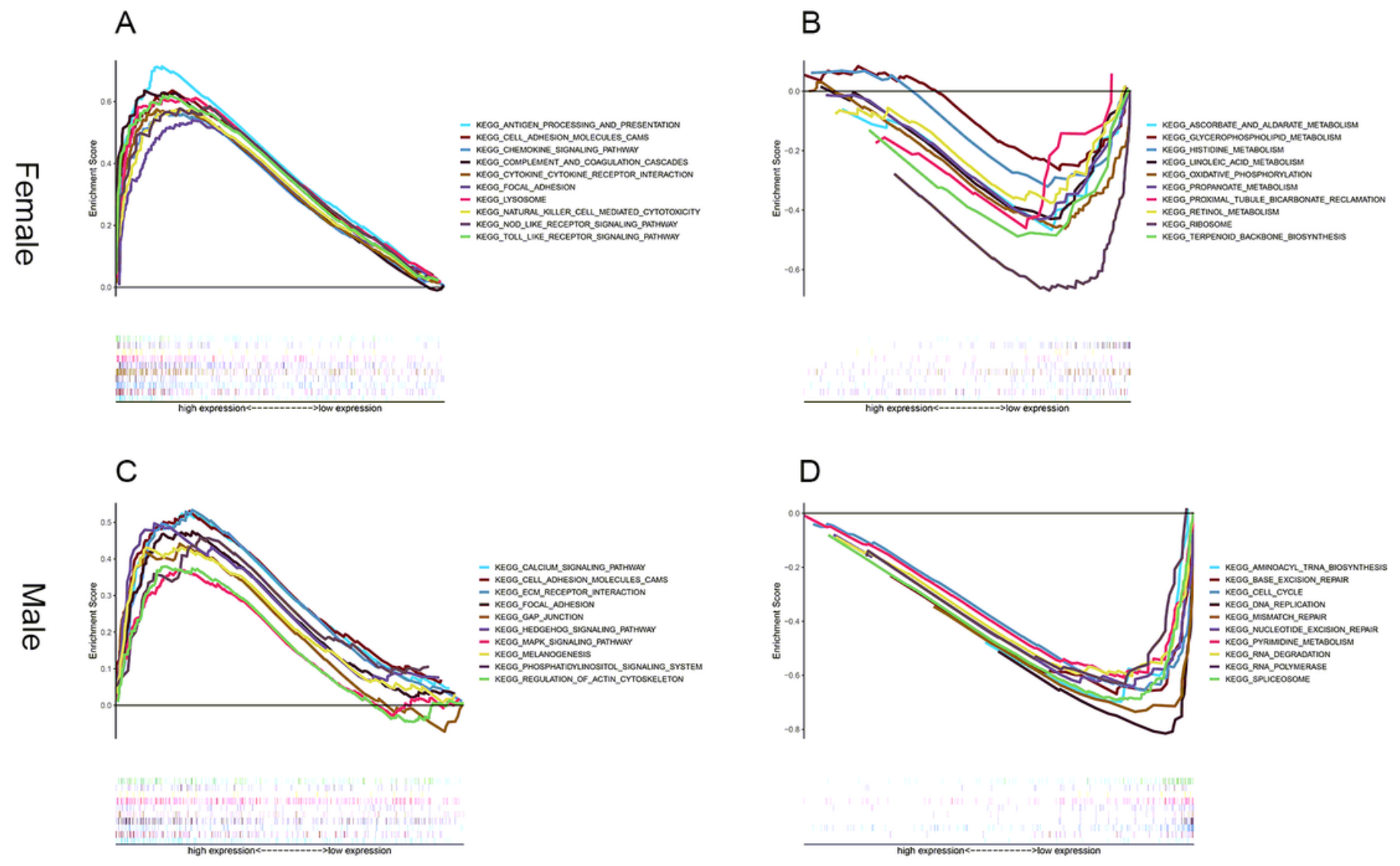

Figure 8

GSEA for samples with high expression and low expression of FCGR2A and GFRA1. The enriched gene sets in the HALLMARK collection by the high (A) and low (B) FCGR2A expression samples. The enriched gene sets in the HALLMARK collection by the high (C) and low (D) GFRA1 expression samples. Each line represents one particular gene set with a unique colour, and upregulated genes are located on the left approaching the origin of the coordinates; by contrast, the downregulated genes are on the right of the $x-$ axis. Only gene sets with NOM $p<0.05$ and FDR $q<0.06$ were to be considered significant. Only several leading gene sets are displayed in the plot. 

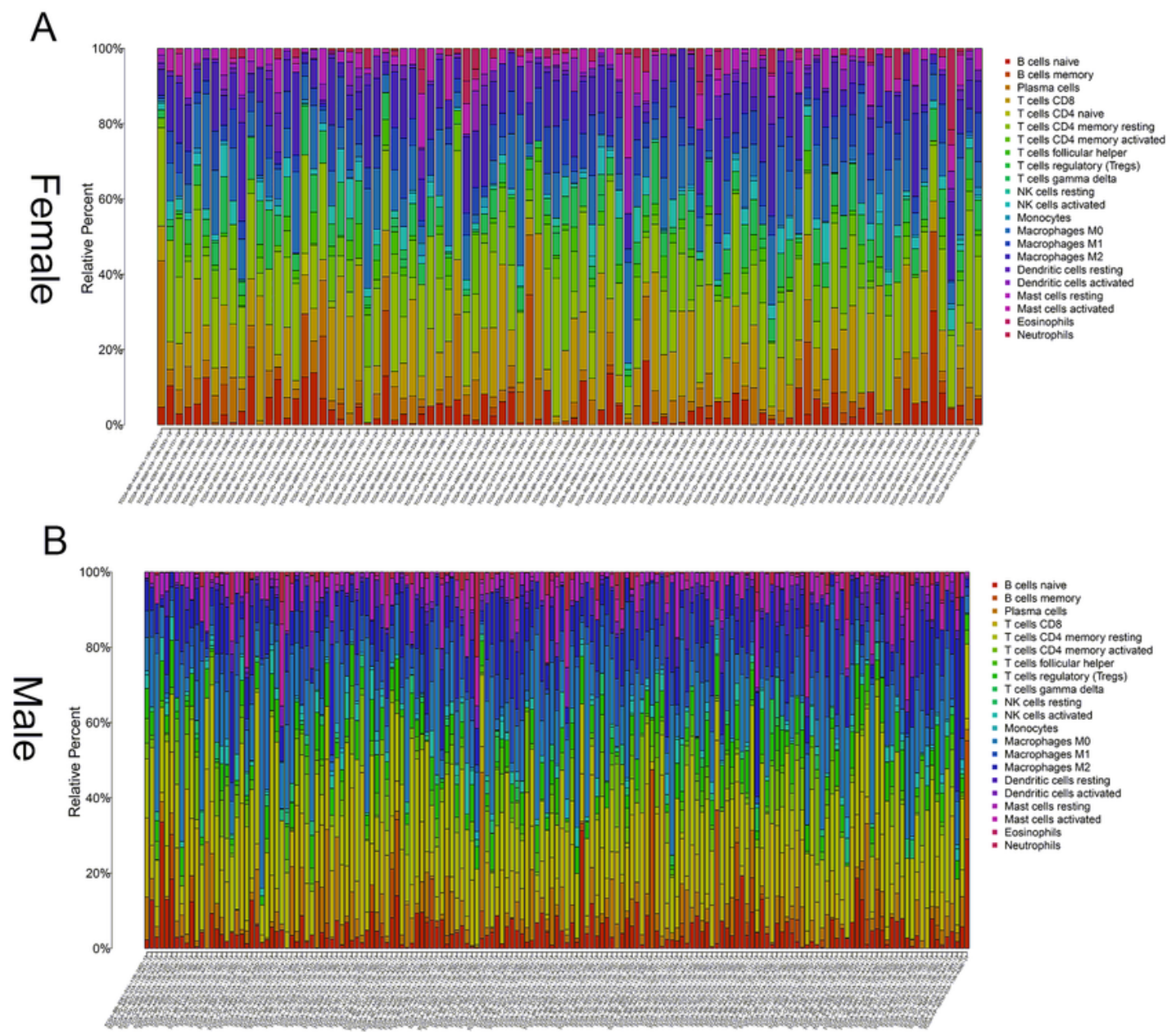

C Female

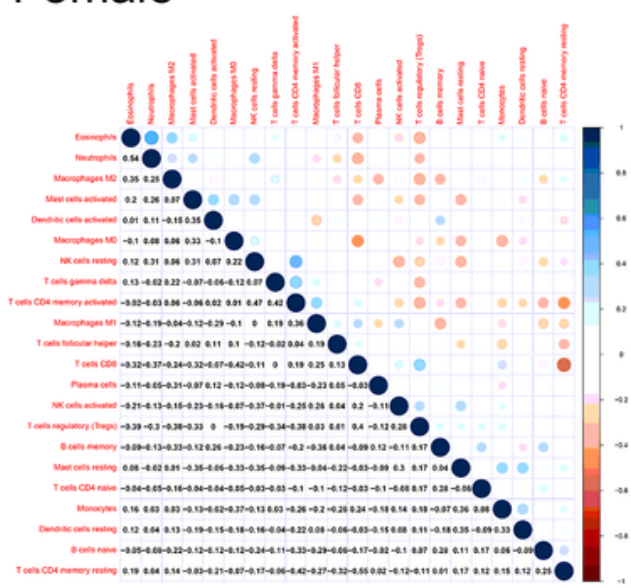

D Male

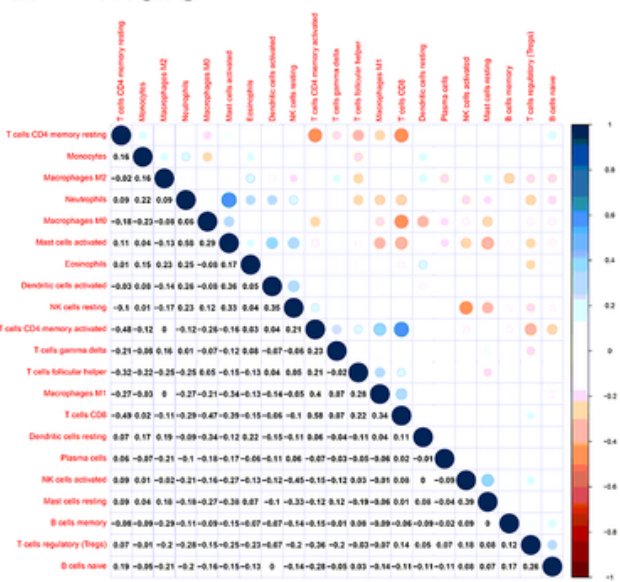

\section{Figure 9}

TIC profile in tumour samples and correlation analysis. Barplot showing the proportion of 22 kinds of TICs in female (A) and 21 kinds of TICs in male (B) GC samples. Column names of plot were sample ID. Heatmap showing the correlation between TICs and numeric cells in each tiny box indicating the $p$ value of the correlation between two kinds of cells in female (C) and male (D) GC patients. The shade of each 
tiny colour box represents the corresponding correlation value between two cells, and the Pearson coefficient was used for the significance test.

A

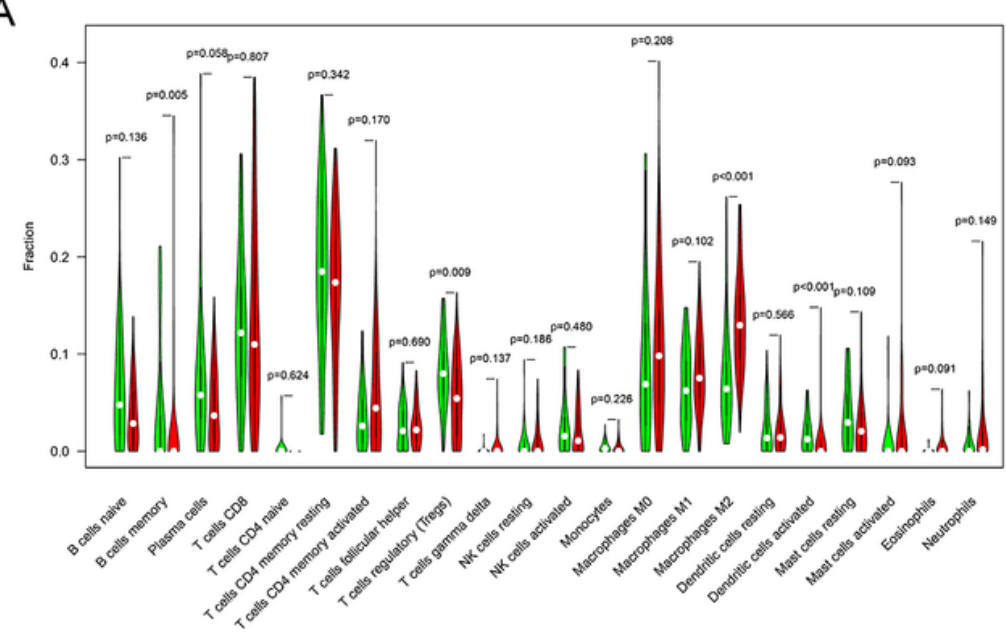

C

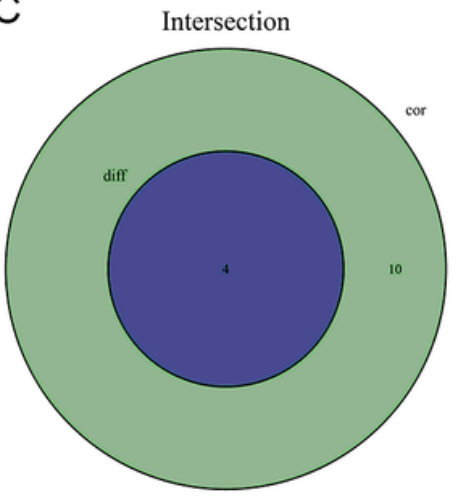

C
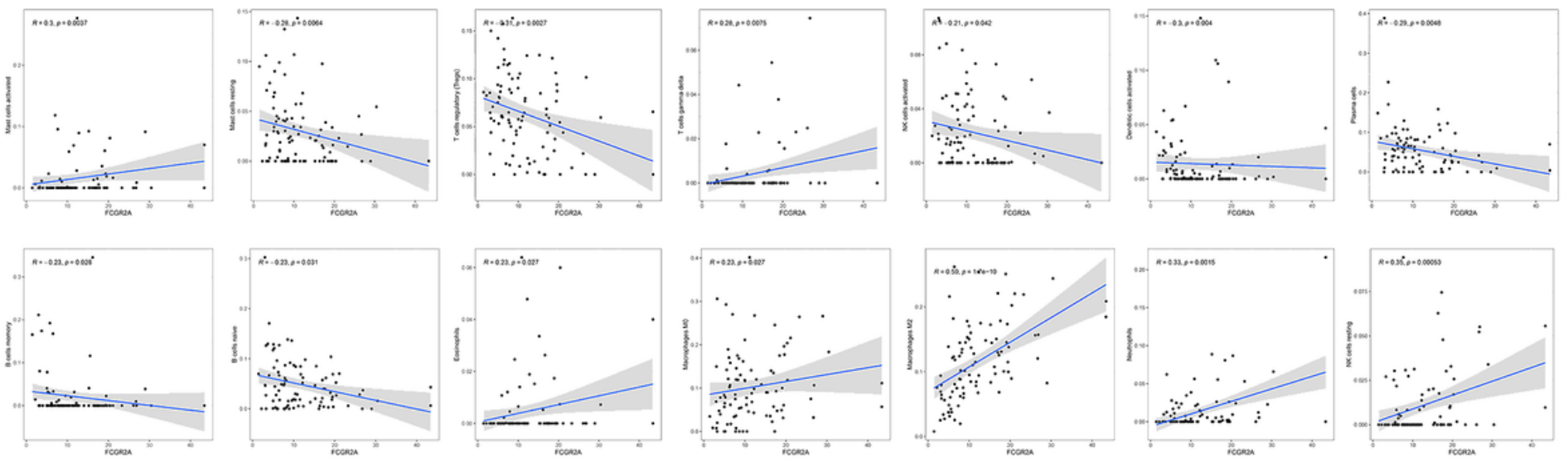

\section{Figure 10}

Correlation of TIC proportion with FCGR2A expression in female GC patients. (A) Violin plot showing the ratio differentiation of 22 kinds of immune cells between GC samples with low or high FCGR2A expression relative to the median FCGR2A expression level, and Wilcoxon rank sum was used for the significance test. (B) Scatter plot showing the correlation of 14 kinds of TIC proportions with FCGR2A expression $(p<0.05)$. The blue line in each plot was fitted to a linear model indicating the proportion tropism of the immune cells along with FCGR2A expression, and the Pearson coefficient was used for the correlation test. (C) Venn plot displaying four kinds of TICs correlated with FCGR2A expression codetermined by difference and correlation tests displayed in violin and scatter plots, respectively. 
A

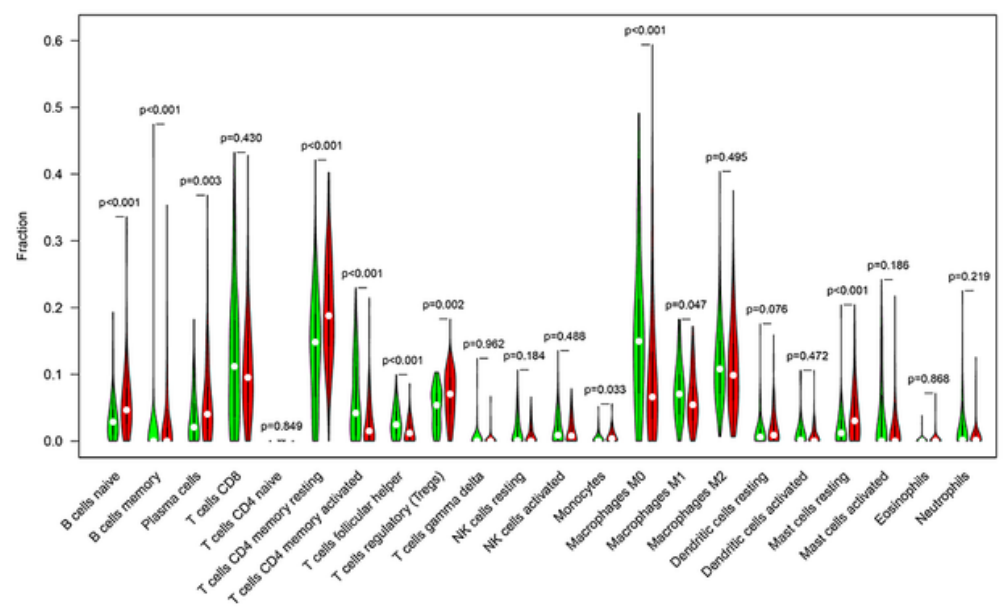

C

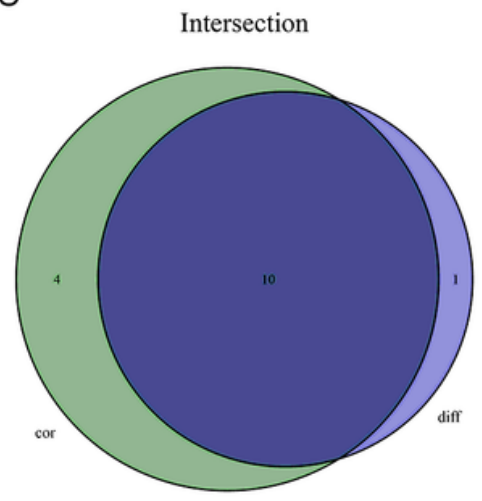

B
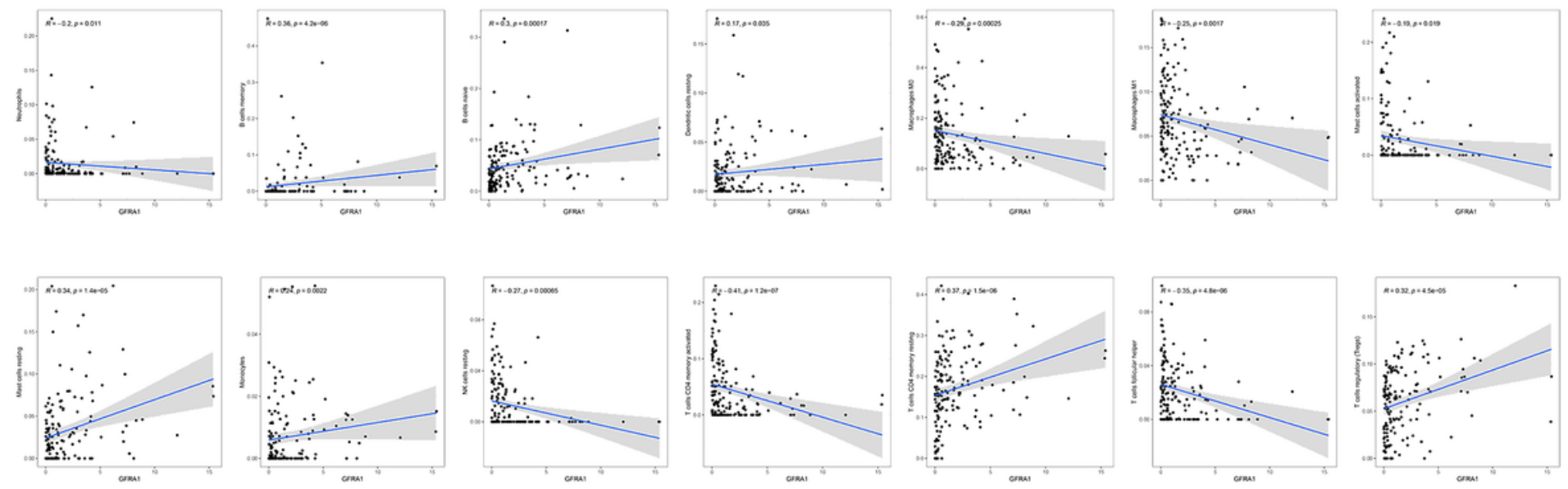

\section{Figure 11}

Correlation of TIC proportion with GFRA1 expression in male GC patients. (A) Violin plot showing the ratio differentiation of 21 kinds of immune cells between GC samples with low or high GFRA1 expression relative to the median GFRA1 expression level, and Wilcoxon rank sum was used for the significance test. (B) Scatter plot showing the correlation of 14 TIC proportions with GFRA1 expression $(p<0.05)$. The blue line in each plot was fitted to a linear model indicating the proportion tropism of the immune cells along with GFRA1 expression, and the Pearson coefficient was used for the correlation test. (C) Venn plot displaying ten kinds of TICs correlated with GFRA1 expression codetermined by difference and correlation tests displayed in violin and scatter plots, respectively. 